\title{
Peripheral neuropathies in the upper extremities of paraplegic wheelchair marathon racers
}

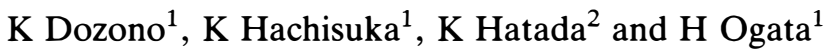 \\ ${ }^{1}$ Department of Rehabilitation Medicine, University of Occupational and Environmental Health, Iseigaoka 1-1, \\ Yahatanishi-ku, Kitakyushu, Fukuoka 807; '2 Oita Nakamura Hospital, Ote-machi 3-2-43, Oita 870, Japan
}

In order to know if peripheral neuropathies in the upper extremities of paraplegic individuals are induced by participating in wheelchair marathon races, we performed nerve conduction studies bilaterally on the median, ulnar, and radial nerves of 10 male wheelchair marathon racers, and on 10 male sedentary paraplegic individuals. In five of 10 paraplegic racers, and in nine of 10 sedentary paraplegics there was a decrease of motor nerve conduction velocities and/or prolongation of motor or sensory nerve distal latencies. Abnormal results in all of the nerve conduction studies were $3.2 \%$ and $13.6 \%$ in paraplegic racers and sedentary paraplegics, respectively. Although paraplegic racers had fewer peripheral neuropathies in the upper extremities than did sedentary paraplegics, ulnar lesions due to involvement of the deep motor branch and at the elbow were a characteristic feature of nerve injuries, presumably caused by wheelchair marathon racing. It is suggested that although a wheelchair marathon is generally a safe sport, wearing gloves and handling a wheelchair in the correct way during the race are necessary to prevent nerve injuries.

Keywords: wheelchair marathon; peripheral nerve injury; upper extremity; paraplegics; spinal cord injury

\section{Introduction}

Nowadays a variety of wheelchair sport tournaments are held around the world, and the wheelchair marathon is one of the established competitions amongst sport activities for the disabled. The wheelchair marathon is not only an enjoyable sport for the disabled, but it is also a way by which they can keep physically fit and obtain a feeling of fulfilment in life., ${ }^{1,2}$ The races, however, are becoming more and more competitive due to better quality wheelchairs, improved training and driving techniques and because of prizes that now include money. Therefore participants must train more and more vigorously to be successful in today's marathon races.

Curtis and Dillon ${ }^{3}$ reported that soft tissue injuries, blisters, lacerations, and arthritis are major complications that can occur in wheelchair athletes and that road racing is one of the high risk activities that can result in a sport injury. Those who have been chronic paraplegic for a long time were also reported to have peripheral neuropathies involving the upper extremities ${ }^{4-7}$ such as the carpal tunnel syndrome and ulnar nerve palsy. Although we suspect that the wheelchair marathon race can cause a paraplegic person to have nerve injuries, we have come across no secondary nerve injuries resulting from wheelchair marathon racing. We therefore performed nerve conduction studies on the upper extremities of paraplegic wheelchair marathon racers and on sedentary paraplegic individuals to investigate whether paraplegic racers have peripheral neuropathy in the upper extremities and if so, what is the feature of such a neuropathy?

\section{Subjects}

Ten male paraplegic marathon racers (PRs), 10 male sedentary paraplegics as paraplegic controls (PCs), and 10 healthy volunteers as controls (HCs) were examined for this study.

The PRs were full time workers in an electrical company, and they enjoyed sports activities; these were established by a welfare corporation in Oita, Japan. The average age was $34.0 \pm 4.9$ years old (mean $\pm \mathrm{SD}$ ), the functional levels were from T8 to L1, and two, and eight of the PRs had complete and incomplete paralysis, respectively. The interval from the spinal cord injury was $11.8 \pm 5.7$ years. The PRs were not elite but were ordinary wheelchair marathon racers, and had enjoyed this type of competition for 7.3 years on average. Seven racers sometimes participated in other sports events such as basketball and tennis. The PRs, who were unable to walk and were bound to their wheelchairs for $12-16 \mathrm{~h}$ a day, usually trained for the wheelchair marathon race for more than $6 \mathrm{~h}$ a week after finishing work.

The PCs were also full time workers, who did not like sports activities; they worked in another electrical company in Fukuoka, Japan. This company was one of the facilities established by the Ministry of Labour of the National Government as a workers' accident 
compensation insurance plan. Their average age was $34.4 \pm 7.2$ years, and their functional levels were from $\mathrm{T} 2$ to cauda equina. The period after the spinal cord injury was $5.8 \pm 3.9$ years. The PCs were bound to their wheelchair during the daytime, and had a sedentary type of life.

The HCs were staff members at the University of Occupational and Environmental Health, Kitakyushu, Japan, with an average age of $33.8 \pm 3.6$ years. They did not have any neurological diseases.

The average ages did not show any significant difference among the PRs, PCs, and HCs by Tukey's multiple comparison test. The time spent and amount of labour was the same in the two electrical companies.

\section{Methods}

The paraplegic marathon racers and the sedentary paraplegics were briefly interviewed regarding subjective symptoms such as muscle weakness, paraesthesia, and the way of manoeuvering the wheelchair. A neurological examination was performed which included manual muscle tests of the abductor pollicis brevis muscle and the first dorsal interosseus muscle, a grip power test, observation of muscle atrophy, two point discrimination and Semmes monofilament tests, Phalen test, and a test to determine percussion pain over nerves.

Nerve conduction studies were done by using a NEC SYNAX ER1100 evoked potential machine (Nippon Electronics Company, Tokyo) in an air-conditioned room. The skin temperature on the right volar forearm was monitored with a Nihon Kohden (Tokyo) electric thermometer and both forearms were kept at $33.5-35.0^{\circ} \mathrm{C}$ with an electric heater during the examination.

The median nerve conduction study consisted of the motor nerve distal latency to the abductor pollicis brevis muscle, motor nerve conduction velocity of the forearm, sensory nerve distal latency from the wrist to the index finger, and sensory nerve latency between the palm and wrist. The ulnar nerve conduction study contained the motor nerve velocities of the forearm and the elbow, motor nerve distal latencies to the abductor digiti minimi muscle and the first dorsal interosseus muscle, sensory nerve distal latency from the wrist to the little finger, and sensory nerve latency between the palm and wrist. The sensory distal latency from the wrist to the thumb was evaluated for the radial nerve conduction study. The precise methods were based on Kimura's textbook (Table 1). ${ }^{8}$

Normal values that were derived from the reports by Kimura, ${ }^{8}$ Davidoff, ${ }^{4}$ Stevens, ${ }^{9}$ Olney, ${ }^{10}$ and Trojaborg ${ }^{11}$ were used in this study (Table 2 ).

All measurements were carried out 1 month prior to a wheelchair marathon race.

\section{Results}

No person complained of paraesthesiae or dysaesthesiae affecting the upper extremities. One individual in
Table 1 Methods for investigating nerve conduction velocities

\begin{tabular}{|c|c|c|}
\hline & Recording & Stimulation \\
\hline \multicolumn{3}{|l|}{ Motor } \\
\hline Median nerve & $\begin{array}{l}\text { Abductor pollicis } \\
\text { brevis muscle }\end{array}$ & $\begin{array}{l}\text { Wrist } \\
\text { Elbow }\end{array}$ \\
\hline \multirow[t]{2}{*}{ Ulnar nerve } & $\begin{array}{l}\text { Abductor digiti } \\
\text { minimi muscle }\end{array}$ & $\begin{array}{l}\text { Wrist } \\
\text { Above elbow } \\
\text { Below elbow }\end{array}$ \\
\hline & $\begin{array}{l}\text { First dorsal } \\
\text { interosseus muscle }\end{array}$ & Wrist \\
\hline \multicolumn{3}{|l|}{ Sensory } \\
\hline Median nerve & Index finger & $\begin{array}{l}\text { Wrist } \\
\text { Palm }\end{array}$ \\
\hline Ulnar nerve & Little finger & $\begin{array}{l}\text { Wrist } \\
\text { Palm }\end{array}$ \\
\hline Radial nerve & Thumb & Wrist \\
\hline
\end{tabular}

Table 2 Normal values of motor and sensory nerve conduction studies

\section{Motor}

Median nerve

Distal latency $(7 \mathrm{~cm})$

Conduction velocity

$2.3-4.6 \mathrm{~ms}$

$49-74 \mathrm{~ms}^{-1}$

Ulnar nerve

Distal latency $(7 \mathrm{~cm})$

$1.8-3.4 \mathrm{~ms}$

Deep motor branch latency between

wrist to adbuctor digiti minimi and

from wrist to first dorsal interosseus muscle $<2.0 \mathrm{~ms}$

Conduction velocity

Forearm

Elbow, compared to forearm

$49-69 \mathrm{~ms}$

$>80 \%$

Sensory

Median nerve latency

Index finger to wrist $(14 \mathrm{~cm}) \quad 2.5-3.5 \mathrm{~ms}$

Palm to wrist $(8 \mathrm{~cm})$

$1.1-1.8 \mathrm{~ms}$

Ulnar nerve latency

Little finger to wrist $(14 \mathrm{~cm}) \quad 1.9-3.1 \mathrm{~ms}$

Palm to wrist $(8 \mathrm{~cm})$

$0.7-1.6 \mathrm{~ms}$

Radial nerve latency

Thumb to wrist $(14 \mathrm{~cm}) \quad 2.0-3.0 \mathrm{~ms}$

the PR group complained of discomfort at the right elbow joint. One each of the PRs and the PCs had a slightly weak abductor digiti minimi muscle; and two of the PRs showed a slightly weak first dorsal interosseus muscle. Neither sensory deficit, a positive Phalen test, nor percussion pain at the nerves were encountered (Table 3).

Table 4 demonstrates the number of injured nerves. In the PR group, five out of the 10 paraplegics had peripheral neuropathies in the upper extremities, while nine out of the 10 paraplegics in the PC group were found to have them. Seven out of all 220 tests $(3.2 \%)$ in the PR group and 30 out of 220 tests $(13.6 \%)$ in the PC group showed abnormal conduction results. The incidence of nerve injuries was higher in the PC group than in the PR group. Concerning the motor nerves, two people in the PR group had a prolonged difference of 
Table 3 Physical examinations

\begin{tabular}{|c|c|c|c|}
\hline & $\begin{array}{l}P R \\
\text { group }\end{array}$ & $\begin{array}{l}P C \\
\text { group }\end{array}$ & $\begin{array}{l}\text { HC } \\
\text { group }\end{array}$ \\
\hline $\begin{array}{l}\text { Subjective symptoms } \\
\text { Discomfort at elbow }\end{array}$ & 1 & 0 & 0 \\
\hline $\begin{array}{l}\text { Objective symptoms } \\
\text { Muscle weakness }\end{array}$ & & & \\
\hline Abductor digiti minimi muscle & 1 & 1 & 0 \\
\hline First dorsal interosseus muscle & 2 & 0 & 0 \\
\hline Sensory deficit & 0 & 0 & 0 \\
\hline Positive Phalen test & 0 & 0 & 0 \\
\hline Percussion pain at a nerve & 0 & 0 & 0 \\
\hline
\end{tabular}

$\mathrm{PR}=$ paraplegic marathon racers $; \mathrm{PC}=$ paraplegic controls; $\mathrm{HC}=$ healthy controls

Table 4 Number of patients and extremities with peripheral nerve injuries

\begin{tabular}{|c|c|c|c|}
\hline & $\begin{array}{c}P R \\
\text { group }\end{array}$ & $\begin{array}{c}P C \\
\text { group }\end{array}$ & $\begin{array}{c}H C \\
\text { group }\end{array}$ \\
\hline \multicolumn{4}{|l|}{ Motor } \\
\hline \multicolumn{4}{|l|}{ Median nerve } \\
\hline Distal latency & $1(1)$ & $4(6)$ & 0 \\
\hline Conduction velocity & 0 & 0 & 0 \\
\hline \multicolumn{4}{|l|}{ Ulnar nerve } \\
\hline Distal latency & 0 & $6(9)$ & 0 \\
\hline Deep motor branch & $2(2)$ & 0 & 0 \\
\hline \multicolumn{4}{|l|}{ Conduction velocity } \\
\hline Forearm & 0 & 0 & 0 \\
\hline Elbow & $1(1)$ & 0 & 0 \\
\hline \multicolumn{4}{|l|}{ Sensory } \\
\hline \multicolumn{4}{|l|}{ Median nerve } \\
\hline Index finger-wrist & 0 & 0 & 0 \\
\hline Palm-wrist & 0 & $1(1)$ & 0 \\
\hline \multicolumn{4}{|l|}{ Ulnar nerve } \\
\hline Little finger-wrist & $1(1)$ & $4(6)$ & 0 \\
\hline Palm-wrist & 0 & 0 & 0 \\
\hline \multicolumn{4}{|l|}{ Radial nerve } \\
\hline Thumb-wrist & $2(2)$ & $5(8)$ & 0 \\
\hline Total & $\begin{array}{l}5 \text { patients } \\
\text { (7 nerves) }\end{array}$ & $\begin{array}{c}9 \text { patients } \\
\text { (30 nerves) }\end{array}$ & $\begin{array}{l}0 \text { patient } \\
(0 \text { nerve })\end{array}$ \\
\hline
\end{tabular}

the ulnar nerve distal latency between the first dorsal interosseus and the abductor digiti minimi muscles.

Figure 1 shows a delayed distal latency of the deep motor branch of the ulnar nerve from the wrist to the first dorsal interosseus muscle, $2.52 \mathrm{~ms}$ (normal value: less than $2.00 \mathrm{~ms}$ ), and a normal distal latency from the wrist to the abductor digiti minimi muscle. A PR who had some discomfort in the elbow was found to have a prolonged ulnar conduction velocity.

There was no relationship between the incidence of peripheral neuropathies and the time since the occurrence of the spinal cord injury, the level of spinal cord injury, and the experience as a wheelchair marathon racer. There was also no difference in the incidence of peripheral neuropathies between the right and the left upper extremities.

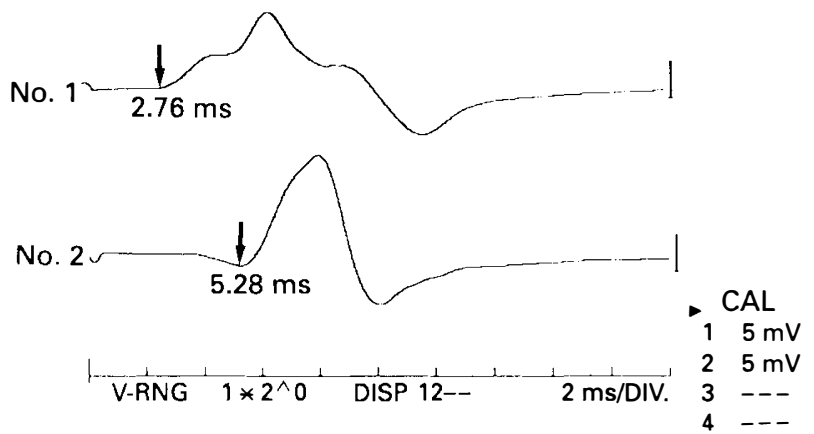

Figure 1 Distal latencies of the ulnar nerve. Arrows indicate the onset latencies from wrist to abductor digiti minimi muscle (No. 1) and from wrist to first dorsal interosseus muscle (No. 2). The difference is $2.52 \mathrm{~ms}$, which means a prolonged latency of the deep motor branch of the ulnar nerve

\section{Discussion}

A wheelchair marathon race held in 1974 at Ohio in the United States of America, was the first such wheelchair sports competition. Ten disabled men took part. It was questionable at that time whether a wheelchair marathon was a suitable sport for the spinal paralysed disabled, but based subsequently on the accumulation of data, the marathon has proven to be very useful for paraplegic individuals as it helps to maintain physical fitness and establishes social relationships.

Secondary injuries due to incorrect and hard training of those participating in wheelchair sports must not be overlooked. Curtis et $a l^{3}$ surveyed wheelchair athletic injuries in 128 paraplegic persons, and reported that there were 291 injuries, made up of 33 soft tissue injuries, 18 blisters, 17 lacerations, and seven pressure ulcers. Track, basketball, and road racing were considered to be high risk sports. Nilson et $\mathrm{l}^{12}$ investigated the risk of paraplegic sportspeople developing complications whilst taking part in sports. Thirty out of 61 competitors developed new complications during the training period. Strains and sprains were the major injuries $(53 \%)$. Ferrara et al ${ }^{13}$ reported that elite wheelchair athletes suffered shoulder $(27.6 \%)$, wrist $(20.7 \%)$, fingers $(17.2 \%)$, hands $(13.8 \%)$, and elbow $(13.8 \%)$ injuries.

On the contrary, long term paraplegic individuals are said to have a predilection for peripheral neuropathies of the upper extremities. Gellman et $a l^{5}$ stated that $49 \%$ of 77 paraplegic patients were observed to have signs and symptoms of a carpal tunnel syndrome, and they speculated that the increasing pressure during the forced extension of the wrist and the repetitive trauma to the volar aspect of the extended wrist whilst propelling a wheelchair may be the cause of this injury. Aljure et $a l^{6}$ also reported that $40 \%$ of 47 long term paraplegic people had a carpal tunnel syndrome. Ulnar nerve injuries, nerve conduction velocity from the mid-arm to below the elbow, and below the elbow to the wrist were significantly low among spinal cord injury patients. ${ }^{7}$ Davidoff $e t a^{4}$ investigated compressive neuropathies of the upper extremities in long term 
paraplegic people and found that $67 \%$ of 32 patients had mononeuropathies. Seventeen $(55 \%)$ had injuries of the median nerve at the wrist, and two had ulnar nerve injuries at the wrist, four at the elbow, and one in the palm of each hand.

The incidence of peripheral neuropathy was $90 \%$ among 'ordinary paraplegic people' in our study, which was higher than that reported in Davidoff's study. ${ }^{4}$ Compared with their study, injuries to the ulnar nerve and radial nerve were more prominent among our non-athlete paraplegics.

In contrast, in our study, $50 \%$ of wheelchair marathon racers had neuropathies. The incidence of peripheral neuropathies of athletes was lower than that of 'ordinary paraplegics', and fewer neuropathies occurred in our racers than in those reported on in Davidoff's study. ${ }^{4}$

Daily training for the wheelchair marathon developed the muscle strength of the upper extremities and kept the body slim. We thought that wheelchair marathon racers had less overload at their wrists in daily activities.

Infrequent but specific injuries to competitors were ulnar nerve neuropathies in the palm and at the elbow. Two racers had injuries of the deep motor branch and one suffered an elbow injury. Davidoff demonstrated compressive mononeuropathies affecting the deep motor branch and the ulnar nerve at the elbow, but he did not indicate the relationship between sports and nerve injury.

Deep motor branch lesions have been observed in cyclists, weight-lifters, installers of plate glass, and in many other occupations. ${ }^{14-16}$ Chronic nerve compression was one of the major causes of these neuropathies. Anatomically, the deep motor branch loops around the hook of the hamate and enters the space between the origin of the abductor digiti minimi and the flexor digiti minimi muscle, giving off branches to each muscle. It then pierces the substance of the opponens digiti minimi muscle and enters the depth of the palm, giving off branches to the lumbricales and the interossei as it crosses the palm. The branch then ends up in the flexor pollicis brevis, the adductors of the thumb and the first dorsal interosseus muscle. According to interviews, most marathon racers handle their wheelchairs with their thumbs or thenar eminences.

After manoeuvering for a long time, they become tired and may propel with the palms of their hands. The deep motor branch is injured by repetitive strokes to the palms whilst propelling their wheelchairs. This injury corresponds to the type $\mathrm{V}$ lesion of Wu's classification. ${ }^{17}$

Ulnar nerve injury at the elbow is induced by a ganglion, by osteoarthritis, or by a cubitus valgus deformity. Wheelchair racers, by repetitive extension and flexion of the elbow, increase pressure on the cubital tunnel, which is a major cause of injury.

To avoid the injuries discussed in this paper, competitors should wear gloves to protect the palms of their hands and, when propelling their wheelchairs, instead of slapping the handrims they should hold them firmly. Paraplegic individuals must use their upper extremities for ambulation, transfer, and many other activities of daily living. Previous studies have reported that many paraplegic people develop nerve injuries at the wrists. It is difficult to avoid such nerve injuries completely. Our study showed that the peripheral nerves of the upper extremities sustained fewer injuries in athletes than was the case for ordinary paraplegic people. We believe that this is due to the fact that the daily training for wheelchair marathon races decreases their body weight and strengthens their muscles. Consequently, they are able to carry out their daily activities without overload at the wrists.

In conclusion, from the point of view of peripheral neuropathy, wheelchair marathons are a safe sport as long as the competitor wears gloves and handles his wheelchair in a proper way.

\section{References}

1 Okuma $\mathrm{H}$, Ogata $\mathrm{H}$, Hatada $\mathrm{K}$. Transition of physical fitness in wheelchair marathon competitors over several years. Paraplegia 1989; 27: 237-243.

2 Hooker SP, Wells CL. Aerobic power of competitive paraplegic road racers. Paraplegia 1992; 30: 428-436.

3 Curtis KA, Dillon DA. Survey of wheelchair athletic injuries: Common patterns and prevention. Paraplegia 1985; 23: 170-175.

4 Davidoff G, Werner R, Waring W. Compressive mononeuropathies in the upper extremities in chronic paraplegia. Paraplegia 1991; 29: 17-24.

5 Gellman $\mathrm{H}$ et al. Carpal tunnel syndrome in paraplegic patients. J Bone and Joint Surg Am 1988; 70: 517-519.

6 Aljure $\mathrm{J}$ et al. Carpal tunnel syndrome in paraplegic patients. Paraplegia 1985; 23: 182-186.

7 Stefaniwsky L, Bilowit DS, Prasad SS. Reduced motor conduction velocity of the ulnar nerve in spinal cord injured patients. Paraplegia 1980; 18: 21-24.

8 Kimura J. Electrodiagnosis in Diseases of Nerve and Muscle: Principles and Practice. FA Davis Company: Philadelphia, 1983.

9 Stevens JC. The electrodiagnosis of carpal tunnel syndrome. Muscle Nerve 1987; 10: 99-113.

10 Olney RK, Hanson M. Neuropathy at or distal to the wrist. Muscle Nerve 1988; 11: 828-832.

11 Trojaborg W, Sindrup EH. Motor and sensory conduction in different segments of the radial nerve in normal subjects. $J$ Neurol Neurosurg Psychiatry 1969; 39: 354-359.

12 Nilsen R, Nygaard P, Bjorholt PG. Complications that may occur in those with spinal cord injuries who participate in sports. Paraplegia 1985; 23: 152-158.

13 Ferrara MS, Davis RW. Injuries to elite wheelchair athletes. Paraplegia 1990; 28: 335-341.

14 Noth J, Dietz V, Mauritz. Cyclist's palsy. J Neurol Sci 1980; 47: $111-116$.

15 Strieb EW, Sun SF, Cochran II RM, Leibrock LG. Distal ulnar neuropathy. Clinical and electrophysiologic aspects. Surg Neurol 1985; 23: 281-286.

16 Hougue RE. Compression of the deep palmar branch of the ulnar nerve. Phys Ther 1985; 65: 203-205.

17 Wu J-S, Morris JD, Hogan GR. Ulnar neuropathy at the wrist: Case report and review of literature. Arch Phys Med Rehabil 1985; 66: 785-788. 